\section{Manejo paliativo de manifestaciones estomatológicas en un paciente pediátrico con leucemia en etapa terminal. Reporte de caso clínico}

\section{RESUMEN}

Los cuidados paliativos en México son programas de asistencia que surgen a partir de la atención de los pacientes con cáncer en la fase terminal de su enfermedad; evitan el sufrimiento del enfermo, aceptan los límites entre el tratamiento curativo y el paliativo, controlan el dolor y atienden los aspectos psicosociales y espirituales. El estomatólogo pediatra debe estar capacitado para poder ofrecer atención de su especialidad como parte del equipo multidisciplinario de cuidados paliativos y eliminar cualquier factor que pueda desencadenar dolor, infección o hemorragia, así como otros síntomas bucales habituales en estos pacientes. Se informa el caso de un paciente pediátrico con leucemia aguda linfoblástica en fase terminal y la intervención de cada uno de los servicios tratantes, incluyendo estomatología, para otorgar al paciente confort y bienestar en los últimos días de vida.

Palabras clave: cuidados paliativos, fase terminal, cuidados de cavidad oral, atención multidisciplinaria.

\section{Palliative Management of oral manifestations in a terminal pediatric patient with Leukemia. Clinical case report}

\author{
ABSTRACT \\ Palliative care in Mexico is based on assistance programs originated \\ from the care of cancer patients in the terminal phase of their illness, \\ directed to prevent suffering, accept the limits between curative and \\ palliative treatment, pain control and attend the psychosocial and \\ spiritual aspects. The pediatric dentistry is responsible for providing \\ dental care as part of a multidisciplinary palliative care team and \\ eliminate any factor that causes pain, infection or bleeding, and \\ other common oral symptoms in these patients. We present the case \\ of a pediatric patient with lymphoblastic leukemia in terminal phase \\ and the intervention of each member of the multidisciplinary team
}

Lorena Guadalupe Rivera-Flores ${ }^{1}$

Eduardo de la Teja-Ángeles

Luis Américo Durán-Gutiérrez ${ }^{3}$

${ }^{1}$ Residente de segundo año de la especialidad de Estomatología Pediátrica.

2 Jefe del Servicio de Estomatología Pediátrica.

${ }^{3}$ Estomatólogo Pediatra Adjunto al Servicio de Estomatología Pediátrica.

Instituto Nacional de Pediatría, Ciudad de México.
Recibido: 8 de abril del 2014

Aceptado: 5 de septiembre del 2014

Correspondencia: Dra. Lorena Guadalupe Rivera Flores

cdlorena_river@outlook.com

Este artículo debe citarse como

Rivera-Flores LG, de la Teja-Ángeles E, DuránGutiérrez LA. Manejo paliativo de manifestaciones estomatológicas en un paciente pediátrico con leucemia en etapa terminal. Reporte de caso clínico. Acta Pediatr Mex 2015;36:97-104. 
including stomatology to provide comfort and improving the quality of life in the last days of life.

Key words: Palliative care, terminal phase, care of mouth, multidisciplinary approach.

\section{INTRODUCCIÓN}

La Organización Mundial de la Salud define los cuidados paliativos como la atención activa de los pacientes con enfermedades que no responden al tratamiento curativo para mejorar su calidad de vida y la de sus familias. ${ }^{1-7}$ Se define a la enfermedad en fase terminal a un padecimiento agudo, subagudo, o crónico de curso inexorable, sin tratamiento curativo posible y sujeto sólo a manejo paliativo. ${ }^{3,4,6,8-10}$

La medicina paliativa tiene una visión integral, responde satisfactoriamente a las diversas necesidades de los pacientes en la fase terminal de su enfermedad y ofrece la posibilidad de morir dignamente..$^{2-4,6,11}$ En México, el cáncer representa un grave y creciente problema de salud pública, constituye una de las principales causas de morbilidad y mortalidad en niños. . $^{2,36}$ Las leucemias son la forma más frecuente de cáncer en pacientes pediátricos, seguidas de los tumores del sistema nervioso central, linfomas, osteosarcomas y retinoblastomas. ${ }^{3,8}$

En el Instituto Nacional de Pediatría, en el periodo del 2010 al 2013, se han atendido por la consulta externa 900 pacientes, y en hospitalización a 377 pacientes, con el diagnóstico de leucemia aguda linfoblástica; de éstos, 52 se consideraron en la etapa terminal de su enfermedad e ingresaron al Programa de Cuidados Paliativos de esta institución.
Los problemas orales son complicaciones comunes de tratamientos contra el cáncer y son muy frecuentes en los pacientes en la fase terminal de su enfermedad ${ }^{12,13-15}$ (Cuadro 1). Es por ello que la presencia del estomatólogo pediatra se hace necesaria en la atención a los pacientes en cuidados paliativos, debido a que pueden existir trastornos, complicaciones o condiciones sistémicas que repercuten directamente en el aparato estomatognático, así como las patologías propias de la cavidad bucal que, en condiciones críticas, son de alto riesgo y complican el tratamiento médico e incluso lo condicionan al fracaso. ${ }^{12,16}$

Las consecuencias de una cavidad bucal insalubre o dolorosa son significativas, no sólo hay consecuencias físicas de la ingesta oral reducida y la pérdida de peso, además puede haber efectos psicológicos debido a la alteración de la comunicación, los sentimientos de exclusión y el aislamiento social. ${ }^{14,15,17,18}$

En general, los tratamientos paliativos se clasifican en:

1. Específicos: son aquellos que varían de acuerdo con la naturaleza propia de cada enfermedad. Ejemplos: cirugía, radioterapia o quimioterapia para control del dolor por tumor óseo; administración de toxina botulínica para el tratamiento de la espasticidad en enfermedades degenerativas. 
Rivera-Flores LG et al. Manejo paliativo en estomatología

Cuadro 1. Síntomas orales frecuentes en pacientes oncológicos en la fase terminal de su enfermedad

\begin{tabular}{|c|c|c|c|c|c|c|c|}
\hline Estudio & $\begin{array}{l}\text { Tipo de población } \\
\text { y tamaño de la } \\
\text { muestra }\end{array}$ & $\begin{array}{c}\text { Boca } \\
\text { seca } \%\end{array}$ & $\begin{array}{c}\text { Malestar } \\
\text { bucal } \\
\%\end{array}$ & $\begin{array}{c}\text { Alteración del } \\
\text { gusto } \\
\%\end{array}$ & $\begin{array}{c}\text { Dificultad para } \\
\text { masticar } \\
\%\end{array}$ & $\begin{array}{c}\text { Dificultad para } \\
\text { deglutir } \\
\%\end{array}$ & $\begin{array}{c}\text { Dificultad } \\
\text { para hablar } \\
\%\end{array}$ \\
\hline $\begin{array}{l}\text { Gordon et al } \\
1985\end{array}$ & $\begin{array}{l}\text { Hospitalizados } \\
\mathrm{N}=30\end{array}$ & 62 & 55 & 31 & 52 & 0 & 59 \\
\hline $\begin{array}{l}\text { Aldred et al } \\
1991\end{array}$ & $\begin{array}{l}\text { Hospitalizados } \\
\mathrm{N}=20\end{array}$ & 58 & 42 & 26 & 0 & 37 & 0 \\
\hline $\begin{array}{l}\text { Jobbins et al } \\
1992\end{array}$ & $\begin{array}{l}\text { Hospitalizados } \\
\mathrm{N}=197\end{array}$ & 77 & 33 & 37 & 0 & 35 & 0 \\
\hline Davies 2000 & $\begin{array}{l}\text { Hospitalizados } \\
\mathrm{N}=120\end{array}$ & 78 & 46 & 44 & 23 & 23 & 31 \\
\hline
\end{tabular}

2. Inespecíficos: aquellas medidas aplicables a cualquier paciente que se encuentra con enfermedad en etapa terminal o condición de vida limitada, sin depender de una etiología particular. Ejemplos: analgesia, tratamiento para la depresión, ansiedad, insomnio, anorexia, infecciones, vómito, estreñimiento, escaras, rehabilitación física, psicológica y social, manejo de otros síntomas y signos orgánicos.

En este sentido, las intervenciones que el estomatólogo realiza pretenden que el paciente recuperé las funciones bucales básicas (masticación, fonación, deglución, succión), mejoré su calidad de vida controlando y eliminando procesos dolorosos, hemorrágicos e infecciosos en tejidos blandos y duros de cavidad bucal y se centran fundamentalmente en que una buena higiene es el aspecto más importante para la integridad oral. ${ }^{12,13,15,18,19}$

En este trabajo se informa el caso de un paciente con leucemia linfoblástica aguda en etapa terminal, con síntomas por complicaciones bucales y su tratamiento.

\section{CASO CLÍNICO}

Niño de 6 años de edad con diagnóstico de base de leucemia aguda linfoblástica pre B de alto riesgo por carga tumoral, detectado en mayo del 2010. Recibió tratamiento de quimioterapia en otra institución; sin embargo, perdió la atención y acudió al Instituto Nacional de Pediatría para continuar su manejo y tratamiento en noviembre del 2011. Al ingreso a este Instituto se encontraba en el mes 17 de remisión completa continua. El Servicio de Hematología inició quimioterapia de reinducción y se detectó recaída a médula ósea. Debido a que el paciente ya presentaba múltiples recaídas durante las quimioterapias y a la elevada carga tumoral, el servicio consideró falla terapéutica y solicitó interconsulta al servicio de Cuidados Paliativos. Dicha unidad evaluó el caso y realizó entrevistas con el familiar a cargo, con el paciente y con los médicos tratantes, los cuales determinaron otorgar el tratamiento paliativo. Se le informó al familiar responsable y se le solicitó la carta de consentimiento informado en la que se indica que el paciente recibiría tratamiento que proporcionara confort y bienestar, contra el dolor, fiebre, procesos infecciosos, sangrado, transfusiones si fueran necesarias, sedante y analgésico a dosis respuesta y se evitaría la intubación orotraqueal y la reanimación cardiopulmonar.

El médico a cargo solicitó interconsulta con el Servicio de Estomatología pues la madre refirió que, al realizar higiene de cavidad bucal con gasa húmeda, notó sangrado en encía, así como 
exfoliación de un órgano dentario. El pediatra refirió que el paciente tenía lesiones en el paladar que habían incrementado de tamaño, con zonas necróticas y diagnóstico probable de aspergilosis. ${ }^{20} \mathrm{~A}$ la exploración física se observó que el paciente presentaba mal estado general y palidez generalizada. En la exploración intraoral el paciente presentó coagulo exofítico (Figura 1), sangrado activo y mucosas pálidas. La biometría hemática reportó hemoglobina $7.2 \mathrm{~g} / \mathrm{dL}$, hematócrito $20 \%$, leucocitos $18200 / \mathrm{mm}^{3}$, neutrófilos $0 / \mathrm{mm}^{3}$, linfocitos $1300 / \mathrm{mm}^{3}$ y plaquetas 49 000/ $\mathrm{mm}^{3}$; tiempos de coagulación con TP 13.9", INR 1.16, TPT 39.4".

Después de la exploración se comentó con el médico responsable que era necesario retirar un coágulo exofítico para determinar origen de la hemorragia, por lo que el Servicio de Hematología decidió trasfundir concentrado plaquetario. Al remover el coágulo exofítico (Figura 2) se encontró el origen del sangrado activo en encía insertada y paladar de incisivo lateral a segundo molar superior derecho, movilidad de incisivo lateral superior derecho y ausencia clínica de primer molar superior derecho, órganos denta-

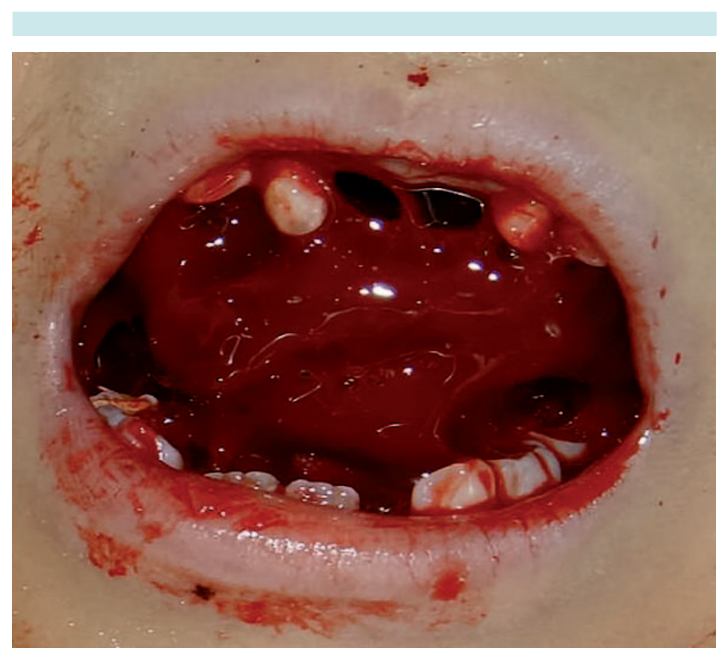

Figura 1. A la exploración intraoral se observa coágulo exofítico relacionado con sangrado en paladar duro del lado derecho.

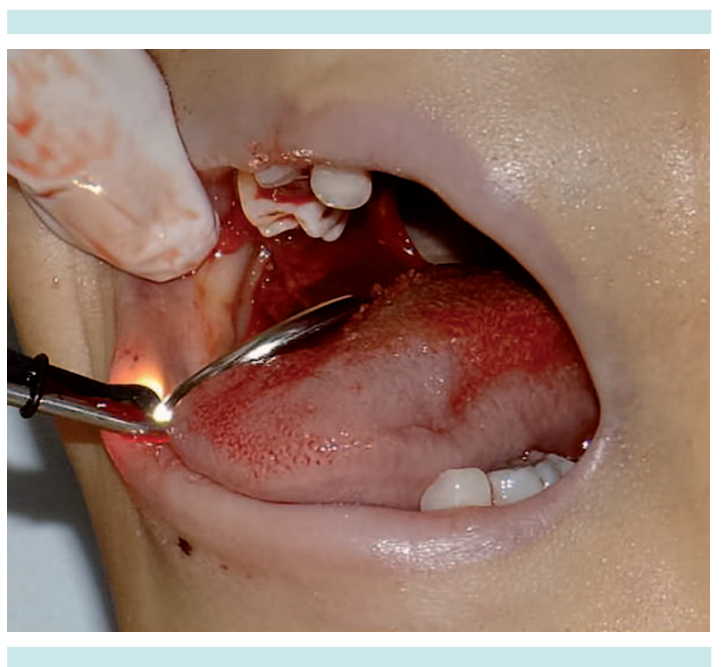

Figura 2. Persistencia de sangrado activo.

rios correspondientes a la formula temporal, por lo que se realizó hemostasia con medios físicos fríos y gasas impregnadas de subsalicilato de bismuto por 8 minutos (Figura 3), con lo que la hemorragia cedió (Figura 4).

Debido a que el paciente no estaba en condiciones sistémicas favorables para realizar biopsia excisional de lesiones necróticas en paladar para determinar la presencia de alguna infección oportunista, se decidió mantener en vigilancia y observación de lesiones y síntomas de la cavidad oral; se dieron medidas generales de hidratación con hielo triturado y humectación constante de labios; dieta blanda en consistencia, fría, libre de irritantes y se instruyó al familiar a cargo para realizar técnica de higiene a base de gasa húmeda; se informó sobre los datos de alarma, principalmente la persistencia de la hemorragia. Se observó la evolución del paciente, para vigilancia de movilidad de incisivo lateral superior derecho temporal y para el soporte y aclaración de dudas del familiar. El paciente se mantuvo asintomático de la cavidad oral.

El servicio tratante indicó administración de antifúngico de forma sistémica para cobertura de 


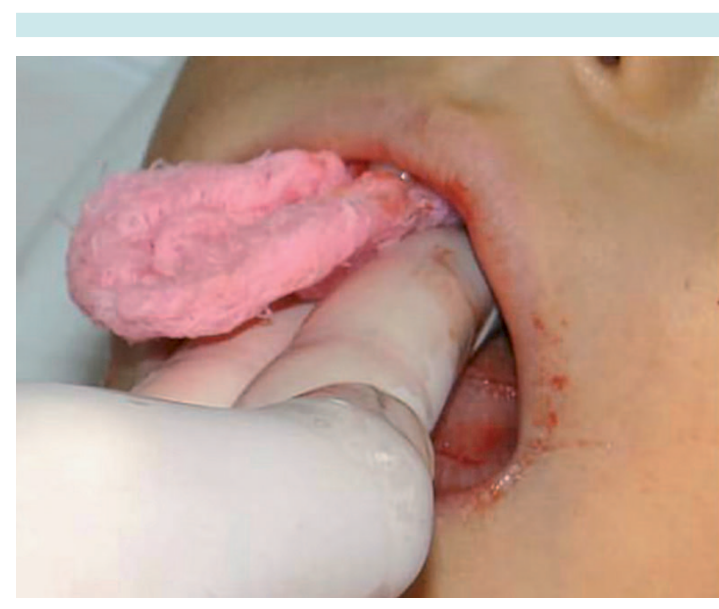

Figura 3. Hemostasia a base de presión y subsalicilato de bismuto después de la remoción de coágulo.

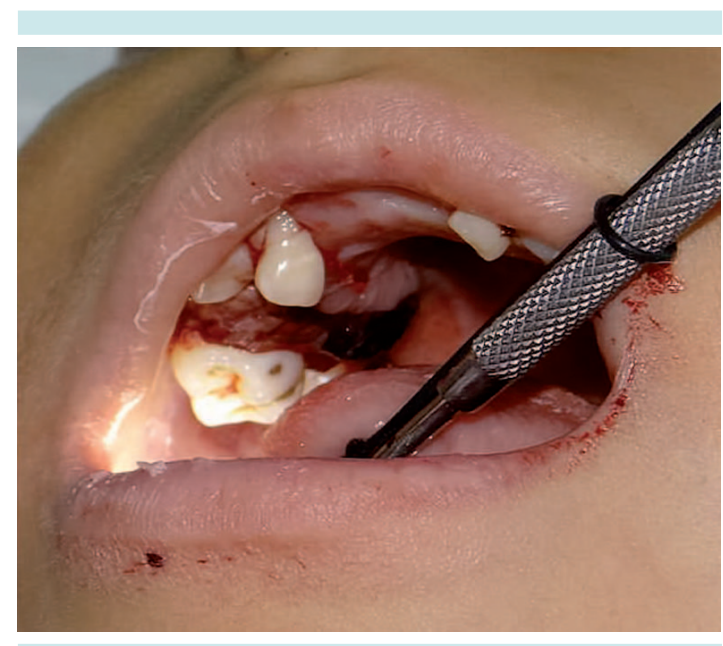

Figura 4. Cese de la hemorragia con el uso de medidas paliativas.

probable infección oportunista. Posteriormente, el menor mostró empeoramiento del estado general, por lo que se indicó sedación, aplicación de oxígeno con mascarilla y morfina; en las horas subsecuentes el paciente falleció. El familiar a cargo se encontró en estado de aceptación y el servicio de cuidados paliativos brindó apoyo emocional.

\section{DISCUSIÓN}

Garduño y sus colaboradores mencionan que la atención de pacientes en la etapa terminal de su enfermedad es multidisciplinaria. ${ }^{1-3,6,8,10} \mathrm{En}$ este artículo se considera que el estomatólogo pediatra debe ser capaz de diagnosticar, tratar y aplicar medidas paliativas para los padecimientos bucodentales en estos pacientes. El manejo estomatológico de pacientes pediátricos en cuidados paliativos consiste en:

1. Manejo hospitalario: en el que se realizan visitas a piso para identificar factores de riesgo que pongan en peligro la integridad bucal, así como la colocación, vigilancia, mantenimiento y uso de dispositivos intraorales auxiliares ${ }^{19}$ y de control de higiene de forma individualizada.

2. Manejo domiciliario: consiste en poder ofrecer atención estomatológica en el hogar del paciente, así como línea telefónica de consulta en caso de emergencia., $3,6,10$

En este caso clínico el familiar a cargo del menor decidió que el paciente se manejara de forma hospitalaria para recibir una vigilancia estrecha de parte de sus médicos; sin embargo, Chirino y Gamboa consideran que si el paciente se estabiliza el programa puede continuar de forma ambulatoria o por visita domiciliaria. ${ }^{4}$

Actualmente existen diferentes sustancias para lograr hemostasia; Reyes y Castro, al igual que Mora y Trujillo, demostraron la seguridad en el uso del subsalicilato de bismuto como agente eficaz en el control de la hemorragia posextracción de terceros molares, ya que permite la liberación de fosfolípidos plaquetarios y actúa directamente en el factor XII de la cascada de coagulación iniciando su vía intrínseca. ${ }^{21,22}$ En este caso se comprobó el efecto hemostático local de las sales de bismuto en tejidos blandos de la cavidad oral en un paciente inmunocomprometido. 
Recomendaciones para la atención estomatológica

Dolor

El punto clave para el éxito en el manejo del dolor es la correcta identificación de la causa (origen dental o de tejidos blandos), así como determinar su intensidad, localización y tolerancia a la vía oral. La evaluación de cada caso de forma individualizada es fundamental para decidir la intervención activa para la resolución del problema de forma radical (drenajes quirúrgicos, tratamientos periodontales quirúrgicos, extracciones) o el manejo meramente paliativo (control del dolor de manera tópica o sistémica), de afectaciones de tejidos blandos, frecuentes en estos pacientes, que incluyen la xerostomía, mucositis y úlceras orales. Algunas de las medidas paliativas que se pueden aplicar son: cambios en la consistencia y temperatura de la dieta, hidratación constante mediante sorbos de agua o de pequeños trozos de hielo, modificación de la técnica de higiene mediante uso de cepillos con cerdas suaves y gasa húmeda, empleo de enjuagues bucales sin alcohol, uso de agentes antisépticos, lubricación y humectación constante de los labios con petrolato puro; aplicación tópica de solución a base de hidróxido de aluminio, hidróxido de magnesio y dimeticona en suspensión con difenhidramina, colocación de dispositivos intraorales auxiliares (protectores bucales, guardas) y, en tejidos duros, la aplicación de odontología de mínima invasión y materiales remineralizantes, así como considerar el uso de analgésicos. ${ }^{12,16,13,14,17,19,23}$

\section{Infección}

En caso de que el paciente curse con algún proceso infeccioso en la cavidad oral se debe determinar la causa para tener un correcto diagnóstico y valorar la eliminación ponderando el riesgo que se toma, así como el beneficio que se obtendrá con el tratamiento, que puede ser radical (extracción) o sólo con medidas paliati- vas (por ejemplo drenaje de abscesos), uso de antibióticos, antifúngicos, antiinflamatorios y analgésicos a dosis respuesta. ${ }^{12,16,13,14,17,19,23}$

\section{Hemorragia}

En caso de hemorragia en la cavidad oral se debe identificar la causa y determinar la localización y extensión de la misma. Es importante la evaluación de estudios complementarios de laboratorio como biometría hemática y tiempos de coagulación, así como el estado general del paciente para determinar si la hemorragia debe ser manejada sistémicamente en conjunto con el médico tratante mediante el empleo de transfusiones. Los métodos locales son: medios físicos fríos (presión mediante gasas húmedas, uso de subsalicilato de bismuto) o, en caso de ser necesario, el empleo de hemostáticos locales (espuma de gelatina, celulosa oxidada) o colocación de puntos de sutura. En caso de sangrados profusos e incontrolables es indispensable usar compresas de color azul o verde, al limpiar la sangre, para disminuir el impacto del color rojo sobre la sábana blanca que impone en los pacientes y familiares, así como mantenerlos informados en todo momento acerca de los procedimientos que se realizarán. ${ }^{12-14,16,17,19,23}$

\section{Centro de atención telefónica}

El manejo domiciliario consiste en ofrecer atención estomatológica en la cama del paciente, así como proporcionar al familiar a cargo una guía informativa para los cuidados de la cavidad oral en casa, donde se señalan las principales manifestaciones estomatológicas en los pacientes con enfermedad en fase terminal y las posibles medidas paliativas que pueden ser útiles para el control de las mismas. Se cuenta con una línea telefónica de apoyo a familiares en caso de emergencia o duda, la cual es atendida por un estomatólogo pediatra capacitado, quien se encarga de brindar y guiar a los familiares a través de sus dudas o 
inquietudes; otorga, paso por paso, las medidas paliativas útiles para cada caso y sobre todo colabora para mantener la calma y disminuir la ansiedad del paciente y de su familia, evitando traslados innecesarios al área de urgencias en situaciones que pueden ser resueltas en casa, o de otro modo programa una visita domiciliaria por parte del estomatólogo pediatra visitador. . $, 6,8,11$

\section{CONCLUSIONES}

El desarrollo de los cuidados paliativos pediátricos (incluyendo al área de estomatología) y una adecuada comprensión de los mismos son necesarios para la atención digna que requiere un paciente con enfermedad en fase terminal. Los cuidados paliativos por parte de la estomatología pediátrica proveen alivio del dolor y otros síntomas; se dirigen principalmente a proporcionar el mayor confort posible y colaboran para lograr la alimentación de forma natural a complacencia de los niños con enfermedad en fase final, para que el paciente disfrute en la medida de lo posible de sus alimentos.

El estomatólogo pediatra ofrece un sistema de soporte para ayudar al paciente en estado terminal a vivir lo más activo posible, instruye y capacita a los padres y cuidadores en el mantenimiento de la salud bucal y acompaña a los niños y a sus familias en uno de los aspectos más profundos de la vida, que es el proceso de morir.

\section{Agradecimiento}

Al Dr. Armando Garduño Espinosa, Jefe de la Unidad de Cuidados Paliativos del Instituto Nacional de Pediatría, por su valiosa colaboración para la realización de este artículo.

\section{REFERENCIAS}

1. Garduño EA. Ham MO. Atención a los Niños con Cáncer en Etapa Terminal. Rev Mex Pediatr. 2006;73(1):33-38.
2. Garduño EA, Ham MO, Méndez VJ, Niembro ZA, Pando CJ, Martínez BR, et al. Decisiones Médicas al Final de la Vida. Recomendaciones para la Atención a Pacientes con Enfermedades en Estado Terminal. Acta Pediatr Mex 2006;27(5):307-16.

3. Garduño EA, Ham MO, Cruz CA, Díaz GE, Reyes LC. Decisiones Médicas al Final de la Vida de los niños. Bol Med Hosp Infant Mex 2010;(67):281-292.

4. Chirino B, Gamboa M. Cuidados Paliativos en Pediatría. Bol Med Hosp Infant Mex 2010;(67):270-280.

5. González C, Méndez J, Romero J, Bustamente J, Castro R, Jiménez M. Cuidados Paliativos en México. Rev Med Hosp Gen Mex 2012;75(3):173-179.

6. Garduño EA. Cuidados y atención del paciente en la UCP. En: Garduño EA. Cuidados Paliativos en Pediatría. México: Trillas; 2011, pp. 35-53.

7. Theunissen JM, Hoogerbrugge PM, Prins JB, Dassen MJ, Ende $\mathrm{CH}$. Symptoms in the Palliative Phase of Children with Cancer. Pediatr Blood Cáncer 2007;49(2):160-165.

8. Garduño EA, Ham MO, Méndez VJ, Rodríguez GJ, Díaz GE, Reyes LC. Experiencias en Cuidados Paliativos en el Instituto Nacional de Pediatría. Rev Mex Pediatr 2009;76(2):75-80.

9. Salazar GT, Méndez XG, Ponce GG, Garduño OC, Muerte Digna o Ensañamiento Terapéutico: una Reflexión para Profesionales de la Salud. Revista Enfermería Universitaria ENEO-UNAM. 2008;1(5):35-40.

10. Garduño EA. Cuidados Paliativos en niños. Atención a pacientes con enfermedad terminal. Acta Pediatr Mex 2004;25(3)1-3.

11. Cuellar R.A. Unidad Pediátrica de Cuidados Paliativos Instituto Nacional de Pediatría. Acta Pediatr Mex 2009;30(5):239-241.

12. Gillam JL. Gillam DG. The assessment and Implementation of Mouth Care In Palliative Care; A Review. The Journal of The Royal Society for the Promotion of Health 2006;126(1):33-37.

13. Milligan S, McGil M, Sweeney M. Oral Care for People With Advanced Cancer an Evidence, based Protocol International. Journal of Palliative Nursing. 2001;7(9):418-426.

14. Jobbins J, Bagg J, Finlay G. Oral and Dental Disease in Terminally III Cancer Patients. BMJ. 1992;304(6842):1612.

15. Dahlin C. Oral Complications at the End of Life. AJN. 2004;104(7):40-47.

16. Wiseman M. The Treatment of Oral Problems in the PaIliative Patient. JCDA. 2006;72(5):453-458.

17. Couch E, Mead JM, Walsh MM. Oral Health Perceptions of Pediatric Palliative Care Nursing Staff. Int J Palliat Nurs 2013;19(1):9-15.

18. De la Teja AE, Durán GA, Espinosa VL, Ramírez MJ. Manifestaciones estomatológicas de los trastornos sistémicos más frecuentes en el Instituto Nacional de Pediatría. Revisión de la literatura y estadísticas del instituto. Acta Pediatr Mex 2008;29(4):189-99. 
19. De la Teja AE, Zurita BY, Duran GA. Dispositivos Intraorales: Guardas Oclusales y Férulas para Pacientes en Estado Crítico. Experiencia del Instituto Nacional de Pediatría. Acta Pediatr Mex 2012;33(4):191-197.

20. Vázquez TO, Campos RT, López SN, Martínez BI. Aspergilosis Palatina en Pacientes con Leucemia: Tres casos Pediátricos. Revista Mexicana de Patología Clínica 2000;47(2):110-115.

21. Reyes VJ, Castro MJ. Uso del Subsalicilato de bismuto como hemostático postextraccion de terceros molares retenidos. Med Oral 2009;XI(1):19-24.
22. Mora LT, Trujillo FJ, Mora SJ. Eficacia y seguridad de la aplicación de subgalato y subsalicilato de bismuto como agentes hemostáticos después de la extracción quirúrgica de terceros molares. Revista ADM 2003;IX(3):90-94.

23. De la Teja AE, Cadena GA, Téllez RJ, Duran GA. El Pediatra ante las urgencias estomatológicas. Acta Pediatr Mex 2007;28(1):21-27. 